\title{
Extracting (-)-hydroxycitric Acid from Dried Rinds of Garcinia oblongifolia Champ. ex Benth by Using Microwave
}

\author{
Dang Quang Vinh*, Dao Hung Cuong, and Nguyen Thuong ${ }^{\dagger}$ \\ Chemistry faculty, University of pedagogy, University of Danang-459 Ton Duc Thang Street, Danang, Viet Nam. \\ *E-mail:vietquocvinh@gmail.com \\ ${ }^{\dagger}$ Environment faculty-Duy Tan University-209 Phan Thanh Street, Danang, Viet Nam
}

(Received February 28, 2011; Accepted June 29, 2011)

\begin{abstract}
Surveys on the process of extracting acid from dried rinds of Garcinia oblongifolia Champ. ex Benth (G. oblongifolia) using microwave in terms of time, machine power and solid/liquid rate, have resulted in such findings as: the best time allocation for extracting is 25 minutes; machine power works best at level 2 (microwave power is $400 \mathrm{~W}$ ); suitable rate of solid/liquid is 0.071 (approximately $150 \mathrm{~mL}$ solvent per $10 \mathrm{~g}$ of sample). The total amounts acid and (-)-hydroxycitric acid extracted from $100 \mathrm{~g}$ dried rinds of $G$. oblongifolia are $18.592 \mathrm{~g}$ and $10.137 \mathrm{~g}$ respectively. This is the first finding on extraction of (-)-HCA from dried rinds of G. oblongifolia using microwave.
\end{abstract}

Key words: Garcinia oblongifolia, (-)-hydroxycitric acid, Microwave

\section{INTRODUCTION}

Acid (-) hydroxycitric (HCA) is a major component in the leaves as well as the rinds of Garcinia, ${ }^{3}$ G. Cowa $^{2}{ }^{2}$. Cambogia, ${ }^{4,8}$ G. Indica. ${ }^{8}$ These plants are grown quite popular in Southeast Asia, India to use as spice for cooking. HCA helps to prevent the fat accumulation and improve the blood lipid level. HCA will boost the excessive glucose metabolism in the body into lipid, help to prevent the obesity, particularly for overweight people who have diet with too much powdered sugar. HCA not only helps to lose weight, HCA also reduces bad lipids for health as triglyceride, LDL cholesterol, total cholesterol and increases HDL cholesterol, which is a fat, has an effect on protection of vascular.

HCA increases glycogen synthesis, oxidation and burning excessive lipid process. With the HCA users, excessive sugar in the body will not turn into fat, yet will be incorporated into glycogen reserving in liver and muscle. When glycogen level is saturated, under the effect of hydroxycitric acid, sugar surplus will active the conversion of fatty acids into mitochondria for burning to form the energy for cells, reducing the excessive fat stored in body. ${ }^{6,7,9,10}$ The products extracted from the Garcinia for weight loss have been sold on the market commonly.

The methods often used for the extraction of (-)-HCA which are the Soxhlet extraction and the distillation in pressure cooker, often are time consuming and easily convert into lactone because of using high temperature. This makes its biological activeness become disabling. With its cheapness, commonality and easily achieving the capacity as desiring, the microwave energy has become more attractive. Microwave-assisted extraction procedure were investigated to optimize the efficiency of the organic acid extraction include: Phenolic acids, ${ }^{1}$ gallic acid, ${ }^{12}$ aristolochic acid, ${ }^{13}$ ascorbic acid, ${ }^{14}$ cajaninstilbene acid,${ }^{15}$ ferulic acid, ${ }^{16}$ oleanolic acid and ursolic acid. ${ }^{17}$ The study of a new extraction method based on microwave energy is essential to help building a complete process to extract the (-)-hydroxycitric acid from the leaves and rinds G. oblongifolia.

This paper presents the extraction process of (-)-hydroxycitric acid from the rinds of G.oblongifolia using the microwave power and factors affecting the capacity of the process.

\section{MATERIALS AND METHODS}

\section{Raw materials and chemicals}

The rinds of the G. oblongifolia, were used as raw materials, were collected in the Hoa lien, Hoa vang district, Danang city. All solvents and chemicals used for highpressure liquid chromatograph were supplied by Merck. Standard compound was (-)-threo-hydroxycitrate tribasic calcium hydrate, BioChemika, Garcinia cambogia Extract, $\sim 19.5 \%$ as $\mathrm{Ca}$ was provided by Sigma-Aldrich company, the formula was $\mathrm{C}_{12} \mathrm{H}_{10} \mathrm{Ca}_{3} \mathrm{O}_{16} \cdot \mathrm{xH}_{2} \mathrm{O}$, the mass of molecular was 530.43 , threo-hydroxycitric acid is $67.3 \%$. Dis- 


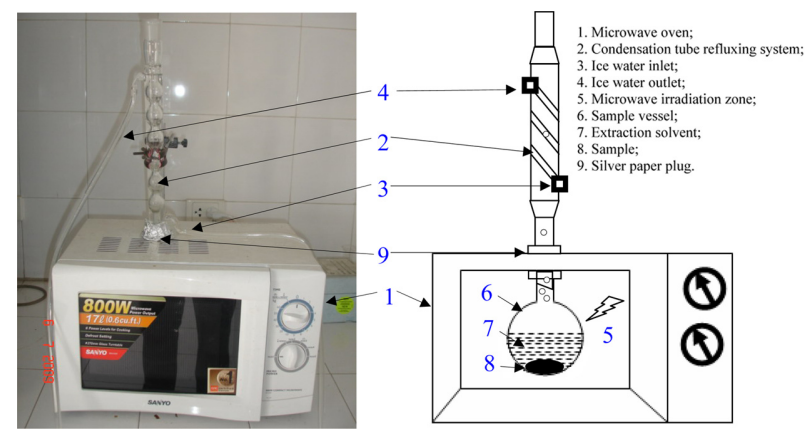

Fig. 1. Extraction system using microwaves.

tilled water was used to extract organic acids and two time distilled water was for HPLC, methanol was used for chromatography, $\mathrm{H}_{3} \mathrm{PO}_{4}$ was $\mathrm{PA}$ type.

\section{Extraction system using microwaves}

Microwave extraction system (Fig. 1) includes the microwave oven and $1000 \mathrm{~mL}$ sample vessel with condensation tube refluxing system. Microwave oven of Sanyo, of which series is EM-S1057 with the following technical parameters: maximum capacity: $800 \mathrm{~W}$, oven volume: 17 litres, external dimension $458 \times 365 \times 295 \mathrm{~mm}$, weight 13.7 $\mathrm{kg}$, power supply $220 \mathrm{~V}, 50 \mathrm{~Hz}$; the furnace with 05 levels corresponding to 05 levels of microwave energy with different capacities, namely: MICRO 1 (80 W), MICRO 2 (400 W), MICRO 3 (500 W), MICRO 4 (650 W), MICRO 5 $(800 \mathrm{~W})$, having button to adjust time and machine level. The maximum time for each operation of the furnace is 60 minutes. ${ }^{11}$

\section{HPLC machine and chromatographic condition}

Chromatographic system: the essential part of Merck Hitachi D7000 consisted of a high-speed auto-sampler, Lichrospher RP18 analytical column $(5 \mu \mathrm{m} \times 4.6 \mathrm{~mm} \times 250$ $\mathrm{mm}$ ), a UV detector set at $210 \mathrm{~nm}$ and Multi - HSM manager software. The mobile phase was phosphoric acid solution $0.1 \%$ with flow rate at $1.0 \mathrm{~mL} / \mathrm{min}$. The standard compound and samples were filtered through $0.45 \mathrm{~mm}$ membrane filter and injected into the HPLC column.

\section{Standard solution Preparation and Calibration standard construction}

Free HCA Preparation: The Calcium threo-hydroxyxitrat tribasic hydrate $(15 \mathrm{mg})$ was treated with $5.0 \mathrm{~mL}$ of water, and with $500 \mathrm{mg}$ Dowex $50\left[\mathrm{H}^{+}\right]$under stirring for 10 minutes using magnetic stirring machine. The liquid part was separated, and ion -exchange resin was washed with water having a neutral $\mathrm{pH}$. The washing water and solution were mixed together and made into $50 \mathrm{~mL}$, followed by mixing, and were filtered using Whatman filter paper 41 .

The construction of the Calibration curve: the construction method of calibration curve was operated based on the analysis results of a series of standard HCA samples. Five standard sample solutions, contained 37.28, 49.70, $62.13,14.55$ and 86.79 ppm of free HCA, were successfully injected into HPLC, The optimal chromatographic condition were done as the above discussion, and the result obtained was the peak area. The HCA curve was drawn based on the dependence between the concentrations of HCA and the peak areas (average of 3 times).

Concentration range of the standard samples needed for the construction of Calibration standards was identified based on the real concentration of HCA in the sample. This concentration range was calculated from the lower limit of quantification (LLOQ) to the upper limit of quantification (ULOQ).

\section{Extraction procedure using microwave energy}

Dried G. oblongifolia rind: After being washed and dried, the G. oblongifolia fruit was then cut to remove nuts and guts. The G. oblongifolia rind was chopped, dried in the oven at $70-80{ }^{\circ} \mathrm{C}$ for 36 hours until the rind was completely dried, powdered and then used for extracting organic acids.

After weighting about $10 \mathrm{~g}$ dried G. oblongifolia rinds, they were put into the distilled water in sample vessel $1000 \mathrm{~mL}$ and it was put into the microwave extraction system (Fig. 1). The main factors selected for the extraction process were extraction time (A) Power (B) and materials ratio $(\mathrm{C})$ (weight of the dried rinds: volume of the extracting agent). The optimum extraction conditions were determined by changing the parameters A, B, C. And then extracts was filtered through muslin cloth. This extracts was brown, opaque. The solution was treated with $4 \mathrm{~g}$ of activated charcoal and heated on a water bath for $30 \mathrm{~min}$. Then, proceeding to filter the extract with buchne funnel and filter paper, activated charcoal was removed continuously by washing two times with $10 \mathrm{~mL}$ of distilled water for each time. The solution treated with activated charcoal became pale yellow. The extract was concentrated to $50 \mathrm{~mL}$, and then treated with $100 \mathrm{~mL}$ of ethanol for 30 minutes to precipitate the remaining pectin (milky white precipitate substance). The floating solution and precipitate compounds were separated using the vacuum filter. After the precipitate part was washed 2 times with $20 \mathrm{~mL}$ ethanol for each time and filtered to collect the 
acid, the filtrate was made up to $100 \mathrm{~mL}$ by the mixing with the floating solution, followed by condensing and adding distilled water. The total acid amount in the sample was determined using acid-base titration method. The sample, after being titrated, was stored at $4{ }^{\circ} \mathrm{C}$ until use for HPLC to determine HCA.

The above experiments were done over times again by changing the parameters A, B, C to examine the capacity of the extraction process based on the microwave irradiation time, the machine level and the ratio of solid / liquid.

\section{RESULTS AND DISCUSSION}

\section{Calibration, Linearity and determination of (-)- HCA by HPLC}

Calibration curve was identified by changing the concentrations of 05 samples at $37.28 ; 49.70 ; 62.13 ; 14.55$ and $86.79 \mathrm{ppm}$, respectively. Equation of calibration linearity was $y=14971 \mathrm{x}-9212.4$, in which $\mathrm{y}$ was the square of HCA peak, $x$ was the concentration of HCA, the correlation coefficient $\mathrm{R}^{2}=0.9997$.

The composition of the organic acid, was determined by high performance liquid chromatography (HPLC) as well as titration method, was shown in Fig. 4, 5, 6. The total contents of acids obtained by acid-base titration process were higher than that of (-)-HCA by HPLC. The major result obtained by HPLC method was HCA, because the peak area of HCA was biggest. After being extracted using microwave energy, the main organic acid was isolated in the G. oblongifolia rind by HPLC was (-)-HCA shown in Fig. 3. According to the resulting chromatography, HCA presents single peak in all of the samples. HCA peaks were identified based on the retention time of standard HCA solution noted at 3.802 min (Fig. 2). The retention time of HCA found in all samples was noted at $3.82 \pm 0.01 \mathrm{~min}$.

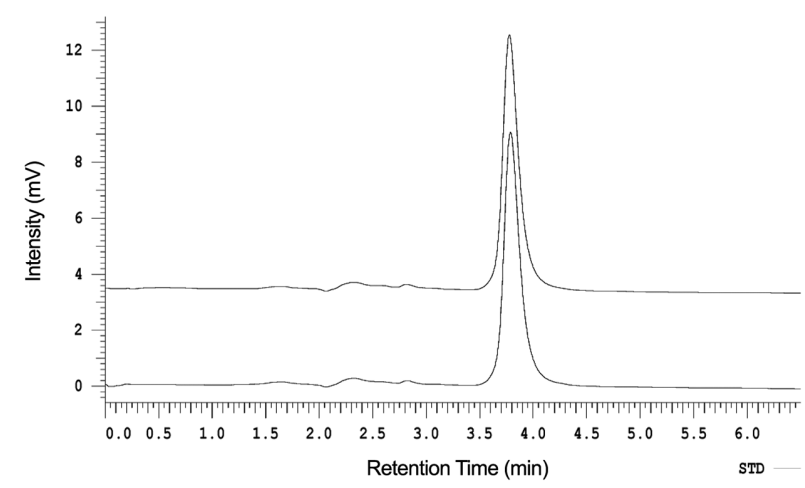

Fig. 2. HPLC Chromatogram of the standard HCA sample.

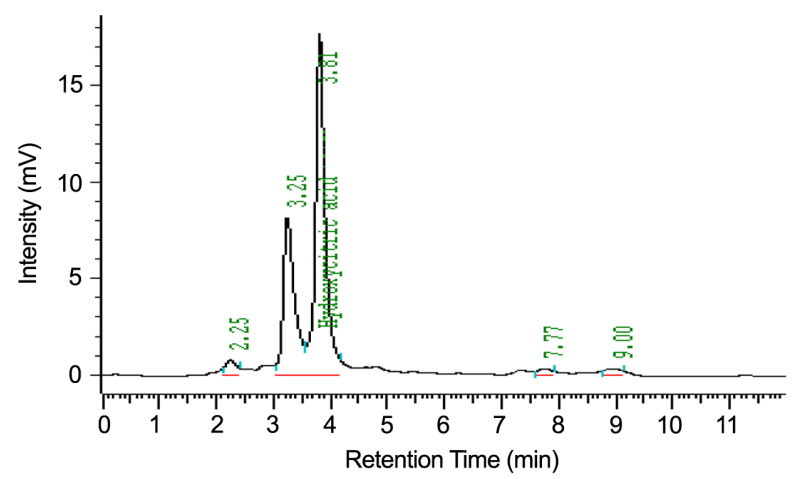

Fig. 3. HPLC Chromatogram of the sample extracted from the dry G. oblongifolia rind with water using microwave power.

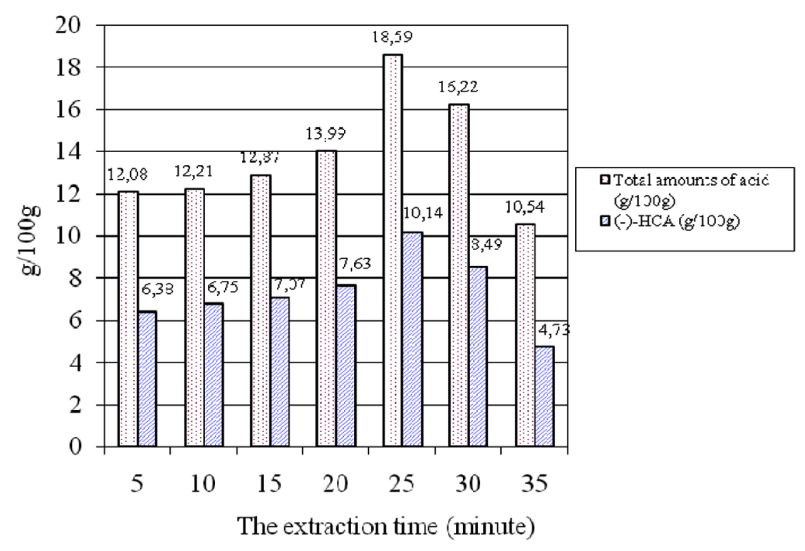

Fig. 4. The dependence of the total amounts of acid and (-)-HCA on the extraction time.

\section{Extraction results over time}

The total amount of organic acid as well as (-)-HCA extracted from the dried rinds of G. oblongifolia using microwave energy, increased with time, was shown in Fig. 4. Fig. 4 indicated that the acid amount raised with the extraction time, reached to the peak at $25 \mathrm{~min}$ (The total amount of organic acid as well as (-)-HCA were $18.592 \mathrm{~g} / 100 \mathrm{~g}$ and $10.137 \mathrm{~g} / 100 \mathrm{~g}$, respectively), followed by the decrease in the amount of acid despite the increase of the extraction time. This can be explained as follows: as the extraction time increases, time for contacting between solvent and the rind of G. oblongifolia fruit raises, so the acid amount extracted boosts. Acids were completely extracted from the G. oblongifolia dried rinds whereas the extract time still remained, acid had risks to be denatured by microwave energy and so the amount of acid decreased.

\section{Extraction result based on the level of machine}

The total amount of acid and (-)-HCA extracted from the $G$. oblongifolia dried rinds using microwave energy, increased with the machine level (microwave intensity), 


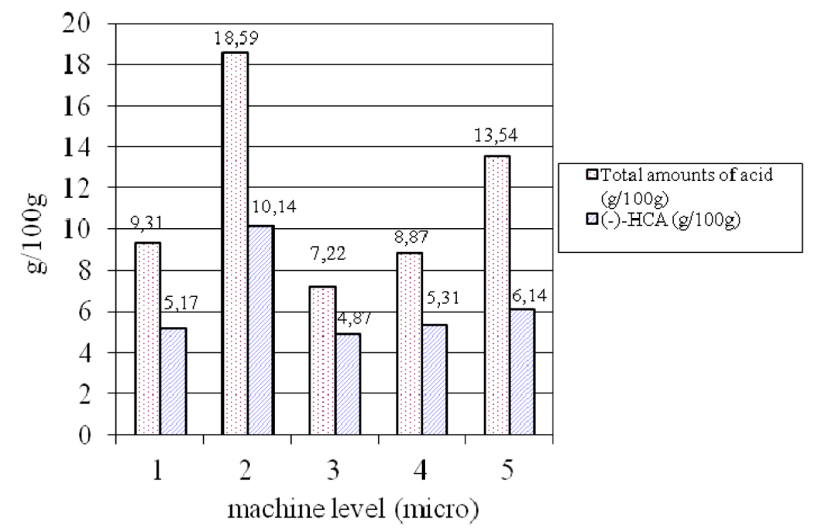

Fig. 5. The dependence of the total amounts of acid and (-)-HCA on the machine level.

were shown in Fig. 5. As raising productivity of machine or machine level, the quantity of acid, in particularly, (-)HCA increased. However, as continuously change the machine level up to micro 3, 4, 5, the acid amount had a tendency to decrease or change insignificantly. As raising the microwave power capacity of machine, that means increasing the machine level, the quantity of acid extracted was more. However, when microwave energy was too large, it can cause the denaturalization of the acid extracted. The suitable power level corresponds to micro $2(400 \mathrm{~W})$ of machine.

\section{Extraction result the ratio of the solid substance and solvent}

The total amounts of the acids and (-)-HCA extracted from the G. oblongifolia dried rinds using the microwave power according to the ratio of the solid substance and solvent were described in Fig. 6. The total acid and (-)-HCA quantity grew gradually with the increase of the volume of solvent or the decrease of the ratio of solid and liquid. The

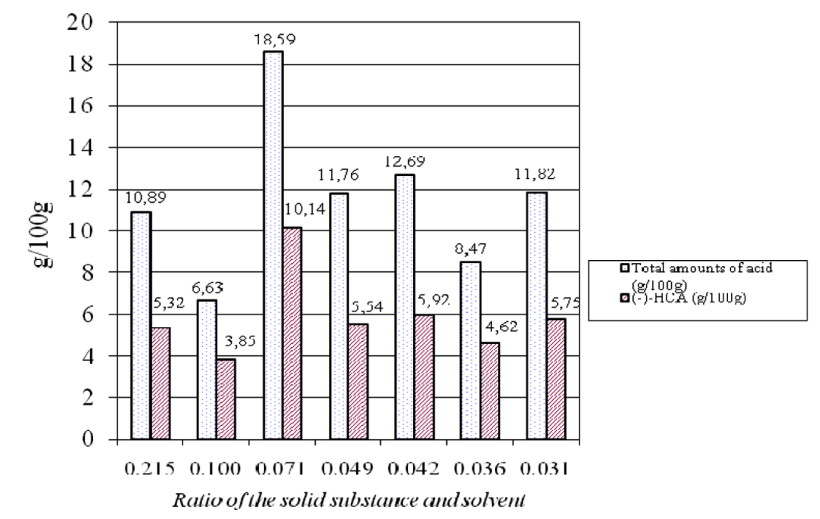

Fig. 6. The dependence of the total amounts of acid and (-)-HCA on the ratio of the solid substance and solvent. acid amount was maximum when the ratio of solid and liquid was 0.071 . And then, although the volume of solvent increased, the total amount of the acids and (-)-HCA decreased and change negligibly. It can be explained as follows: as increasing the solvent volume for each solid quantity, it means that the soluble capacity of acid extracted from solid phase to liquid phase raises, so the acid amount obtained increases. However, because the extraction times (15 min) as well as microwave capacity (400 W) were constant, the microwave energy supplied for the extract (the sample) was invariable. As a result, when the solvent volume increased, at first the amount of acid obtained raised, and then reduced. Because of the increase of the extraction solvent volume, it would take a longer time for boiling of the extract. It results the decrease in the quantity of acid extracted by thermal motion reduced. The ratio of solid and liquid giving the highest result was 0.071 , approximately $150 \mathrm{~mL}$ of solvent for $10 \mathrm{~g}$ dried G. oblongifolia rinds.

\section{CONCLUSION}

In summary, (-)-hydroxycitric acid was extracted from the dried G. oblongifolia dried rinds by using microwave power. The results obtained from the investigation of extracting hydroxycitric acid process from the dried G. oblongifolia rinds using microwave energy according to time, machine level and ratio of solid and liquid showed that: The most effective time for extraction was $25 \mathrm{~min}$; the appropriate machine level was 2 level (microwave power was $400 \mathrm{~W}$ ); the suitable ratio of solid and liquid was $0.071(150 \mathrm{~mL}$ of solvent for $10 \mathrm{~g}$ solid substance approximately); the total amount of organic acids as well as (-)-HCA obtained were $18.592 \mathrm{~g} / 100 \mathrm{~g}$ sample and $10.137 \mathrm{~g} / 100 \mathrm{~g}$ sample, respectively. Under the optimum conditions, microwave-assisted extraction could provide higher extraction yields of total amount of organic acids $(18.592 \mathrm{~g} / 100 \mathrm{~g})$ than Soxhlet extraction $(13.711 \mathrm{~g} / 100 \mathrm{~g})$ and pressure cooker distillation (17.468 g/100 g), higher extraction yields of (-)-HCA than Soxhlet extraction $(9,508 \mathrm{~g} / 100 \mathrm{~g})$ and less (-)-HCA than pressure cooker distillation $(15,221 \mathrm{~g} / 100 \mathrm{~g}){ }^{3}{ }^{3}$ in addition to having a shorter extraction time. Comparing (-)-HCA microwave-assisted extraction from G. oblongifolia with Soxhlet extraction from G. Cowa $(10,209-12,260 \mathrm{~g} / 100 \mathrm{~g}){ }^{2}$ pressure cooker distillation from G. India $(10,27-12,74 \%)^{5}$ and G. cambogia (16-18\%), ${ }^{4}$ these results indicated that (-)-HCA microwave-assisted extraction from G. oblongifolia is an average. 
From this result, it could be concluded that the microwave power can be used for extraction of (-)-HCA from the material of the dried G. oblongifolia dried rinds to produce weight-los products.

\section{REFERENCES}

1. Arunkumar Thandavarayan, Sathishkumar, T.; Shanmugam, S.; Sadasivam, S.; Microwave Assisted Extraction of Phenolic acids from Vitex negundo leaves. Journal of Pharmacy Research 2011, 4(4), 998.

2. Bhabani, S. Jena.; Guddadarangavvanahally. Jayaprakasha, Kunnumpurath K. S. Organic Acids from Leaves, Fruits, and Rinds of Garcinia cowa. Journal of Agricultural and Food chemistry 2002, 50(12), 3431.

3. Dao Hung Cuong, Dang Quang Vinh. Estimating hydroxycitric acid in leaves, rinds, fruits of Garcinia oblongifolia champ. ex benth by HPLC. Journal of Science and Technique - Danang university 2007, 3(20), 137.

4. Jayaprakasha, G. K.; Sakariah, K. K. Determination of organic acids in Garcinia cambogia (Desr.) by HPLC. Journal of Chromatography A 1998, 806(2), 337.

5. Jayaprakasha, G. K.; Sakariah, K. K. Determination of organic acids in leaves and rinds of Garcinia indica (Desr.) by LC. J. Pharm. Biomed. Anal. 2002, 28(2), 379.

6. Jena, B. S.; Jayaprakasha, G. K.; Singh, R. P.; Sakariah, K. K. Chemistry and biochemistry of (-)-hydroxycitric acid from Garcinia. J. Agric. Food Chem. 2002, 50(1), 10.

7. Kriketos, A. D.; Thompson, H. R.; Greene, H.; Hill, J. O. (-)-Hydroxycitric acid does not affect energy expenditure and substrate oxidation in adult males in a post-absorptive state. Int. J. Obes. Relat. Metab. Disord. 1999, 23(8).

8. Lewis, Y. S.; Neelakantan, S. (-)-Hydroxycitric acid - The principal acid in the fruits of Garcinia cambogia. Phytochemistry 1965, 4, 619.

9. Lewis, Y. S. Isolation and properties of hydroxycitric acid, In Methods in Enzymology; Colowick, S. P., Kaplan, N. O., Eds.; Academic Press: New York, 1969; Vol. 13, pp 613-619.

10. Lowenstein, J. M. Effect of (-)-hydroxycitrate on fatty acid synthesis by rat liver in vivo. J. Biol. Chem. 1971, 246(3), 629.

11. Microwave Oven User Guide, Seri EM-S1057; Sanyo Electric Co., LTD.

12. Verma, S. C.; Nigam, S.; Jain, C. L.; Pant, P.; Padhi, M. M. Microwave-assisted extraction of gallic acid in leaves of Eucalyptus xhybrida Maiden and its quantitative determination by HPTLC. Der Chemica Sinica 2011, 2(2), 268.

13. Zhou, T.; Xiao, X.-H.; Wang, J.-Y.; Chen, J.-L.; Xu, X.-F.; He, Z.-F.; Li, G.-K. Evaluation of microwave-assisted extraction for aristolochic acid from Aristolochiae Fructus by chromatographic analysis coupled with nephrotoxicity studies, Biomedical chromatography, BMC Apr 2011.

14. Yu, Y.; Chen, B.; Chen, Y.; Xie, M.; Duan, H.; Li, Y.; Duan, G. Nitrogen-protected microwave-assisted extraction of ascorbic acid from fruit and vegetables. J. Sep. Sci. 2009, 32(23-24), 4227.

15. Yu Kong et al. Optimization of microwave-assisted extraction of cajaninstilbene acid and pinostrobin from pigeonpea leaves followed by RP-HPLC-DAD determination. Journal of Food Composition and Analysis 2010, 23(2010), 382.

16. Zhilan Liu, Wang, J.; Shen, P. N.; Wang, C. Y.; Shen, Y. J. Microwave-assisted extraction and high-speed countercurrent chromatography purification of ferulic acid from Radix Angelicae sinensis. Sep. Purif. Technol. 2006, 52, 18.

17. Xinsheng Fang, Jianhua Wang, Xiaoliang Yu, Guohui Zhang, Jinpeng Zhao. Optimization of microwave-assisted extraction followed by RP-HPLC for the simultaneous determination of oleanolic acid and ursolic acid in the fruits of Chaenomeles sinensis. J. Sep. Sci. 2010, 33(8), 1147. 